Artigo Original

\title{
AVALIAÇÃO DO EXTRATO BRUTO DE ARTEMÍSIA VULGARIS NA RESPOSTA IMUNOLÓGICA
}

\author{
Autor: Lucas S. Zamarioli', Ed Wilson Santos ${ }^{2}$, Maria Cristina Marcucci ${ }^{3}$, Carlos R. Oliveira ${ }^{1, A}$. \\ ${ }^{1}$ Grupo de Fitocomplexos e Sinalização Celular, Escola de Ciências da Saúde, Universidade Anhembi Morumbi, São Paulo, \\ SP 03164-000, Brazil. 'Laboratório de Hematologia Clínica, Faculdade de Ciências Farmacêuticas, Universidade de São \\ Paulo (USP). ${ }^{3}$ Laboratório de Produtos Naturais e Quimiometria, Programa de pós-graduação em Farmácia e Biotecnologia, \\ Universidade Anhanguera de São Paulo, São Paulo, SP.
}

\section{Informações do artigo}

Palavras Chave:

Artemisia vulgaris, Imunomodulação, Etnofarmacologia.

\section{Resumo}

A Artemisia vulgaris é uma das espécies do gênero Artemisia, gênero o qual possui grande história terapêutica e estudos. Suas partes aéreas são tradicionalmente consumidas pela população sob forma de infusão (chá), por acreditar que possui propriedades anti-helmíntica, antisséptica, antiespasmódica, anti-inflamatória e, até mesmo, como tônico para órgãos vitais. Este trabalho, teve como objetivo avaliar o extrato bruto (EB) da Artemisia vulgaris na resposta imunológica, com base na história terapêutica da planta e na etnofarmacologia.

Para o desenvolvimento, foram utilizadas técnicas para obtenção e análise dos compostos do extrato por cromatografia líquida de alta eficiência (HPLC), sendo identificados como os principais compostos a artemisinina, e os ácidos clorogênico e cafeoilquínico. Posteriormente foi realizado o tratamento da linhagem celular RAW (macrófagos murinos) com o EB. As células foram tratadas com diferentes concentrações de extrato (de 0,25\% a $2 \%$ ), por um período de 24 horas, e estimuladas com lipopolissacarídio (LPS) bacteriano, com o intuito de se observar a produção de citocinas. Após a segunda incubação por 24 horas, o sobrenadante dessa linhagem foi recolhido, e a análise da produção de citocinas pró-inflamatórias, interleucina (IL)-6 e anti-inflamatórias, IL-4, foi feita por imunoensaio (teste de ELISA), avaliando, assim, a influência do extrato de A.vulgaris na resposta imunológica.

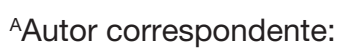

Carlos Rocha Oliveira - e-mail: carlos.oliveira@laureate.com.br - ORCID: https://orcid.org/0000-0001-8634-2850

DOI: https://doi.org/10.31415/bjns.v2i3.60 - Artigo recebido em: 24 de junho de 2019; aceito em 05 de agosto de 2019 ; publicado em 30 de setembro de 2019. Brazilian Journal of Natural Sciences, Vol. 2, N.3, setembro 2019. Disponível online a partir de 30 de setembro de 2019, ISSN 2595-0584. www.bjns.com.br Todos os autores contribuíram igualmente com o artigo. Os autores declaram não haver conflito de interesse. Este é um artigo de acesso aberto sob a licença CC - BY: http://creativecommons.org/licenses/by/4.0 


\section{Article ID}

Keywords:

Artemisia vulgaris, Immunomodulation, Ethnopharmacology.

\begin{abstract}
Artemisia vulgaris is one of species from the Artemisia gender, this gender has a long therapeutic history and studies. Their aerial parts are consumed for the population as tea. People believe that A. vulgaris tea has properties anti-helmintic, antisseptic, anti-spasmodic, anti-inflammatory and even tonic for the vital organs. This study, had the main objective the evaluation of the crude extract of Artemisia vulgaris in the immunologic response, with the therapeutic history and etnopharmacology basis. For the development, technics for the obtention and analysis of the composition of the extract was made by High Performance Liquide Chromatography (HPLC), being detected as main substances, the artemisinin, and chlorogenic and cafeoilquinic acids. Subsequently we made the treatment of the cell line RAW (murine macrophages) with the extract. The cells were treated with different extract concentrations (from $0,25 \%$ to $2 \%$ ), for 24 hours, and stimulated by bacterial lipopolysaccharide (LPS), with intention of observe the citokynes production. After the second incubation, for 24 hours, the supernatant were collected, and the production of pre-inflammatory, interleukin (IL)- 6 and anti-inflammatory, IL-4, cytokines were analyzed by immunoassay (ELISA test), thus evaluating the influence of $A$. vulgaris extract on the immunologic system.
\end{abstract}

\section{Introdução}

A Imunomodulação envolve tanto estímulo, como redução de certas respostas imunes celulares e/ou humorais. Neste sentido, produtos de origem natural, demonstram exercer efeitos imunomoduladores [1]. Por exemplo, pimenta preta (Piper nigrum) e cardamomo (Elettaria cardamomum) apresentaram potentes efeitos imunomoduladores [1]. Produtos naturais com atividade imunomoduladora são amplamente utilizados no tratamento de muitas doenças, incluindo doenças autoimunes, distúrbios inflamatórios, além do câncer [2].

O gênero Artemísia (Artemísia spp.) apresenta uma história terapêutica há mais de dois mil anos, sendo amplamente utilizada na medicina popular, além de ser um dos gêneros mais estudados devido a sua diversidade morfológica, química e biológica [3].As espécies que fazem parte do gênero Artemisia spp., compreendem, na verdade, importantes plantas medicinais que atualmente são objeto de atenção fitoquímica devido à sua produção grande produção de metabólitos secundários, diversidade química e biológica [4]. As espécies do gênero Artemisa spp., apresentam uma gama de atividades biológicas, como no caso da espécie Artemisia annua, que possui polissacarídeos com atividade antitumoral e dotada de propriedades imunomoduladoras [5].

Em trabalho publicado por Hunt [6]. o extrato de Artemisia annua mostrou-se seguro e eficaz no manejo da dor e da limitação funcional associa à osteoartrite presente no quadril e no joelho de pacientes.

Bao et.al. [7], utilizou os polissacarídeos de Artemisia argyi sobre células tumorais verificando a inibição do crescimento do sarcoma 180 (S180) de camundongos.

Estudos in vitro demonstraram a capacidade do extrato de Artemisia absinthium em reduzir os níveis de TNF-alfa e outras interleucinas $[8,9,10$. Também em relação ao extrato de Artemisia absinthium, verificou-se atividade hepatoprotetora conferida à camundongos, através de atividade imunomoduladora e antioxidante desta espécie vegetal [11].

A Artemisia vulgaris, uma das espécies do gênero Artemisia spp, é tradicionalmente utilizada pelas suas propriedades anti-helmíntica, antisséptica, antiespas- 
módica, como tônico para órgãos vitais e outras desordens como a hepatose [12].

Polissacarídeos obtidos das folhas de Artemisia vulgaris, reduziram a proliferação e indução à diferenciação de células leucêmicas humanas THP-1, mostrando que o extrato poderia ser utilizado como tratamento complementar a leucemia mielóide aguda [13]. Além disso, a Artemisia vulgaris apresentou propriedades antioxidantes [12].

Deste modo, este projeto visa avaliar a atividade imunomoduladora do extrato hidroalcoólico de $A r$ temisia vulgaris através do tratamento in vitro de macrófagos murinos, seguido do estímulo por lipopolissacarídio (LPS), a fim de se avaliar, por imunoensaio, as interleucinas 4, 6, 10, 12 e interferon-gama (IL-4, IL-6, IL-10, IL-12 e INF- $\gamma$ ).

Devido ao uso popular de plantas do gênero Artemísia spp, e da potencialidade das plantas medicinais como novas opções terapêuticas, a realização deste trabalho teve como objetivo estudar a possível atividade imunomoduladora da espécie Artemísia vulgaris, a fim de se compreender o mecanismo de interação desta espécie vegetal com o sistema imunológico, e fornecendo, desse modo, informações sobre um possível candidato capaz de conferir aumento da resposta orgânica contra vírus, bactéria, fungos e protozoários, além de poderem exercer um papel importante no auxílio à terapêutica antitumoral, no transplante de órgãos e medula óssea e nas doenças autoimunes.

Sendo assim, este estudo, além do aproveitamento acadêmico com o aprendizado de diferentes metodologias, forneceu mais informações sobre a Artemisia vulgaris, espécie vegetal muito utilizada popularmente.

\section{Objetivos}

Avaliação da capacidade imunomoduladora do extrato hidroalcoólico de Artemisia vulgaris, através do tratamento de macrófagos de camundongos (células RAW).

Avaliação da produção de citocinas pré e pró-inflamatórias das células submetidas ao tratamento com extrato de A. vulgaris.

\section{Material e método}

\section{Reagentes}

Os reagentes que foram utilizados no estudo são: Artemisinina (Sigma-Aldrich), lipopolissacarídio (Escherichia coli 055:B5), meio de cultura DMEM-high e soro fetal bovino (SFB). Imunoensaio com enzima conjugada (ELISA) kits de interleucinas (IL)-4, IL-6, IL-10, IL-12 e interferon (IFN)- $\gamma$.

\section{Obtenção do extrato de Artemisia vulgaris}

O extrato hidroalcoólico de Artemisia vulgaris na forma de tintura mãe homeopática foi preparado seguindo as padronizadas técnicas laboratoriais industrializadas - Laboratório Homeopático Almeida Prado, São Paulo, Brasil. O extrato foi preparado de acordo com a Farmacopeia Homeopática Brasileira e fornecido como um extrato etanólico a $65 \%$. Este extrato inicial foi concentrado sob pressão reduzida a temperaturas inferiores a $45^{\circ} \mathrm{C}$ utilizando um evaporador rotativo (Buchi Rotavapor R-200), e o solvente foi completamente removido.

Análise cromatográfica por cromatografia líquida de alta eficiência (HPLC) - identificação de artemisinina em Artemisia vulgaris

Uma quantidade do extrato de Artemisia vulgaris (com concentração conhecida) foi solubilizada com $5 \mathrm{~mL}$ de metanol a quente, filtrada em papel de filtro e passada por um filtro Millipore $(0,45 \mu \mathrm{m}$, Sartorius) para a análise por cromatografia líquida de alta eficiência em fase reversa (HPLC). A identificação de artemisinina nos extratos foi avaliada por HPLC, empregando-se uma coluna $\mathrm{C} 18$ em fase reversa, por método isocrático, consistindo de metanol e água acidificada com $0,2 \%$ de ácido fórmico (50:50). O fluxo foi de $1,0 \mathrm{~mL} / \mathrm{min}$ e a detecção monitorada em 254 nm. Foi utilizado um detector de rede de diodos na detecção dos picos [14], para verificação de artemisinina em Artemisia vulgaris.

\section{Células}

As células RAW foram gentilmente cedidas pelo Prof. Ed Wilson, do Departamento de Ciências Farmacêuticas da Universidade São Paulo. Foram culti- 
vadas em suspensões contendo $2 \mathrm{~mL}$ de meio DMEM-high, suplementado com 10\% FCS (Hyclone), 4 mM L-glutamina, $80 \mu \mathrm{g} / \mathrm{mL}$ penicilina e $80 \mu \mathrm{g} / \mathrm{ml}$ de estreptomicina (Hyclone), $1 \mathrm{mM}$ de piruvato de sódio (Hyclone), 10 mM HEPES, 1x aminoácidos não-essenciais (Sigma, St. Louis, MO) e $6 \times 10^{5} \mathrm{~m} \beta$-ME].

\section{Tratamento, produção e análise de citocinas}

Para o tratamento, as células foram divididas em 2 grupos, são eles: A) Grupo estimulado com LPS, e B) Grupo controle (não estimulado com LPS). Ambos os grupos foram tratados com o extrato de Artemisia vulgaris em forma de gradiente, começando com $0 \mu \mathrm{L}$ e aumentando gradualmente de $5 \mathrm{em} 5 \mu \mathrm{L}$ até $40 \mu \mathrm{L}$, totalizando 9 wells para cada grupo.

$\mathrm{O}$ experimento ocorreu em três etapas, na primeira, foram adicionadas em cada well $1,5 \times 10^{5}$ células, com $2 \mathrm{~mL}$ de meio DMEM- high suplementado, e os grupos "A" e "B" foram tratados com o extrato e incubados por $24 \mathrm{~h}$ à $36^{\circ} \mathrm{C}$ com atmosfera com $5 \%$ de $\mathrm{CO}_{2}$.

$\mathrm{Na}$ segunda etapa o grupo "A" foi estimulado com $10 \mu \mathrm{g} / \mathrm{mL}$ de LPS, e ambos voltaram a ser incubados nas mesmas condições citadas acima.

A terceira etapa consistiu na coleta do sobrenadante, após a segunda incubação de $24 \mathrm{~h}$, e seu armazenamento à menos $80^{\circ} \mathrm{C}$ antes do ensaio ser realizado. Os níveis de citocinas em sobrenadantes foram medidos por imunoensaio (ELISA) conforme o protocolo do kit e Bioscience e a leitura da placa foi feita pelo aparelho Thermo Scientific 5250030 VARIOSKAN FLASH.

\section{Análises estatísticas}

Os resultados foram apresentados como média + EPM (erro padrão da média). Os resultados obtidos foram submetidos à análise estatística por análise de variância (ANOVA) de uma via, seguido de teste a posteriori de Tukey. As semi-quantificações foram analisadas por test " $\mathrm{t}$ " de Student. Valores de $\mathrm{P}<0,05$ foram considerados significativamente diferentes. As análises foram realizadas no programa GraphPad Prism versão 5.0.

\section{Resultados}

\section{Cromatografia}

Tanto a corrida do padrão de artemisinina quanto à do extrato hidroalcoólico de Artemisia vulgaris ocorreram nas seguintes condições cromatográficas:

Solvente A: água acidificada a $0.1 \%$ com ácido fórmico, e solvente B: metanol. Corrida de 10 minutos, iniciando com $95 \%$ de A à 100\% de B em 8 min, mantendo-se até 8,5 minutos, retornando as condições iniciais e estabilizando até 10 minutos.

temperatura da coluna foi de $40^{\circ} \mathrm{C}$ com volume de injeção de $10 \mu \mathrm{l}$ da solução padrão e das amostras em uma coluna C18, (partícula $1.7 \mu$, tamanho $2.1 \mathrm{~mm} \mathrm{x}$ $50 \mathrm{~mm}$ ). Varredura ESI modo positivo e negativo entre 100 e $700 \mathrm{~m} / z$.

Sendo assim, a figura 1 mostra o cromatograma em UHPLC da tintura de Artemisia obtido em modo positivo, o íon de massa $(\mathrm{m} / \mathrm{z}) 283$, com o tempo de retenção de 5,27 minutos, indicando a presença da Artemisinina.

A figura $2 \mathrm{~A}$ apresenta o cromatograma com melhor resolução indicando o tempo de retenção de 5,27 minutos.

E a figura 2B é o pico com esse tempo de retenção ampliado, para melhor visualização.

\section{Figura 1.}

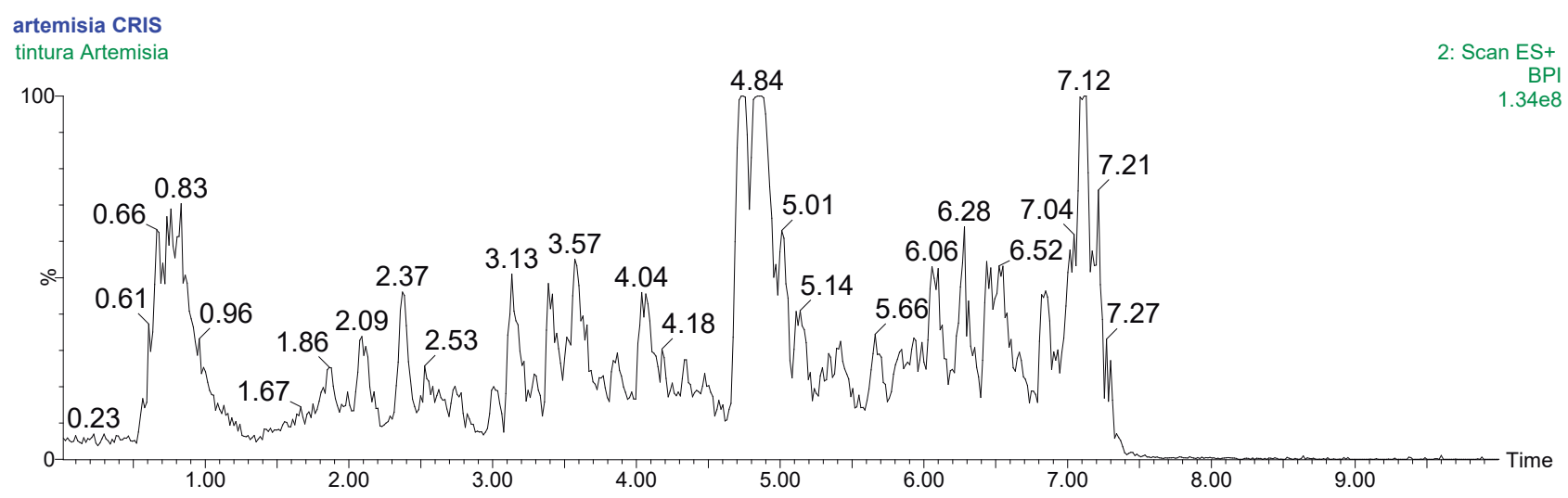




\section{Figura 2A.}

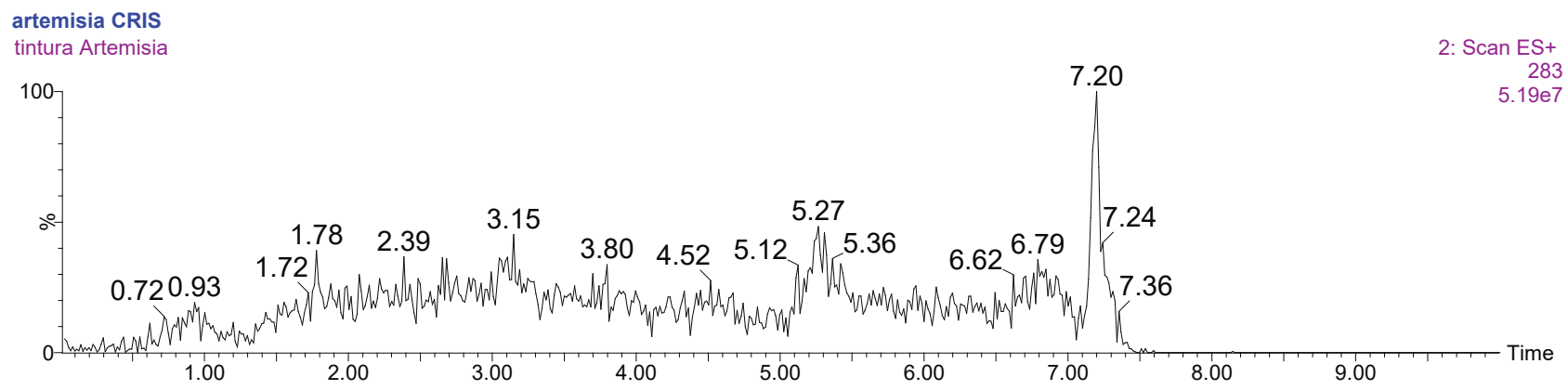

\section{Figura 2B.}

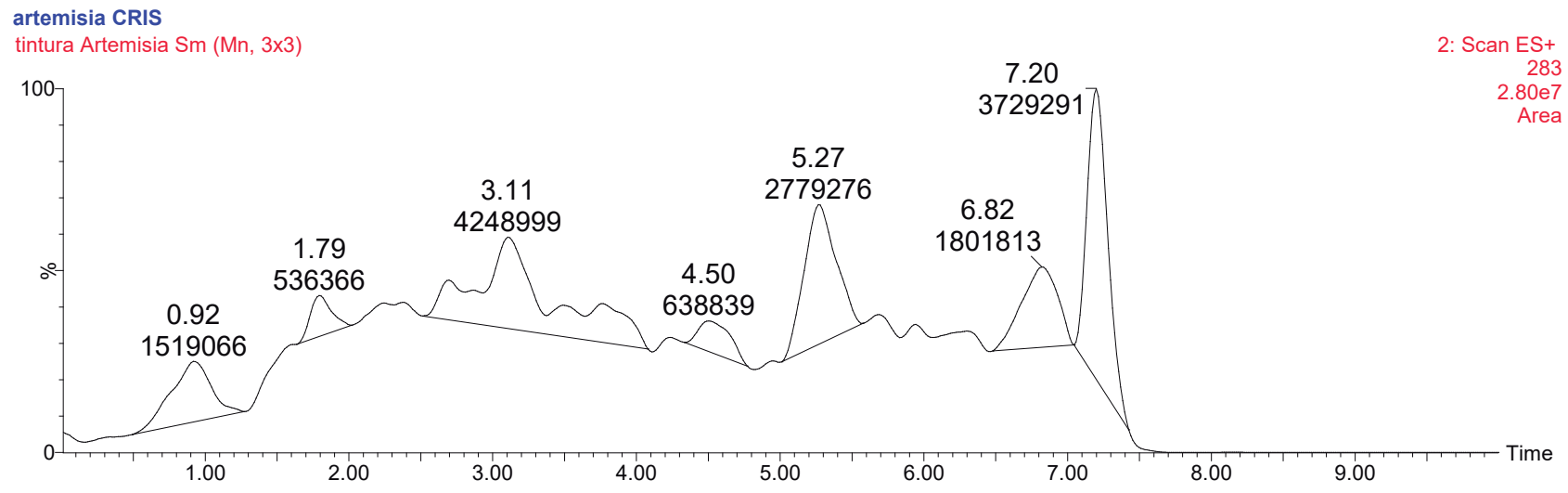

A figura 3 mostra a análise por UHPLC do padrão de artemisinina.

Figura 3.

artemisia CRIS

padrao Artemisinina 2

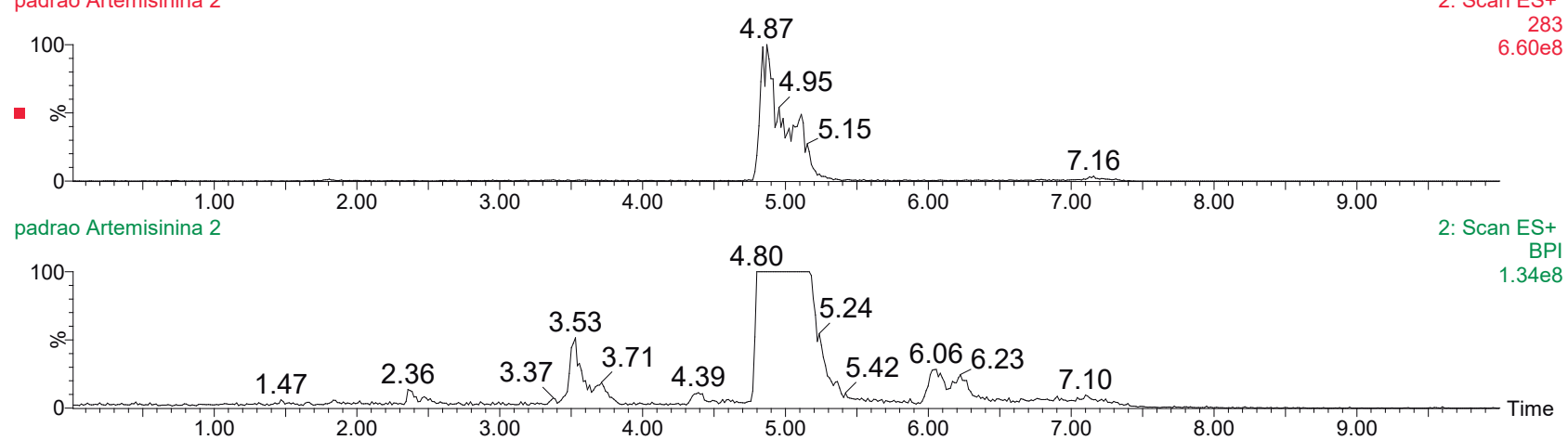

O cromatograma acima está de acordo com os resultados obtidos por Khairul et al [15], indicando o mesmo tempo de retenção do padrão da artemisinina.

A figura 4A mostra o cromatograma por UHPLC da tintura de Artemisia vulgaris em modo negativo. Enquanto $4 \mathrm{~B}$ mostra o tempo de retenção do ácido clorogênico e isômeros em 1,62 e 1,84 minutos. A figura $4 \mathrm{C}$ indica a presença de ácidos cafeoilquinicos e isômeros com o tempo de retenção 2,62 e 2,75 minutos.

Outra corrida foi realizada, desta vez apenas com o extrato de A.vulgaris, com as seguintes condições cromatográficas:

HPLC LaChrom série L7100 com detector de rede diodos série L7455 da Merck e Hitachi (Alemanha). Coluna C18 Lichrocart 125-4mm, 5 micrometros, solvente A água acidificada com ac. Fórmico a 5\% e solvente B metanol. 


\section{Figura 4A.}

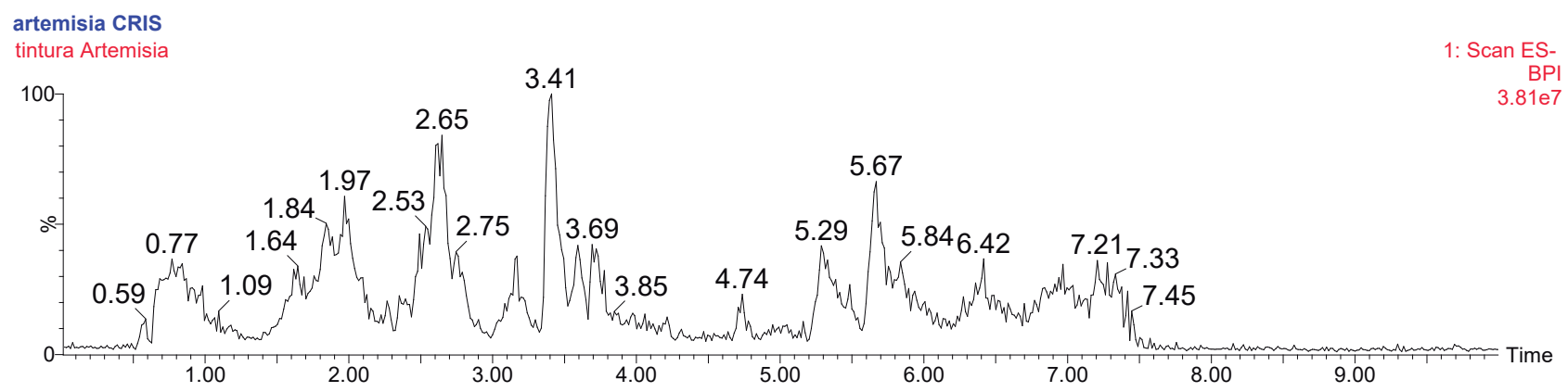

\section{Figura 1B.}

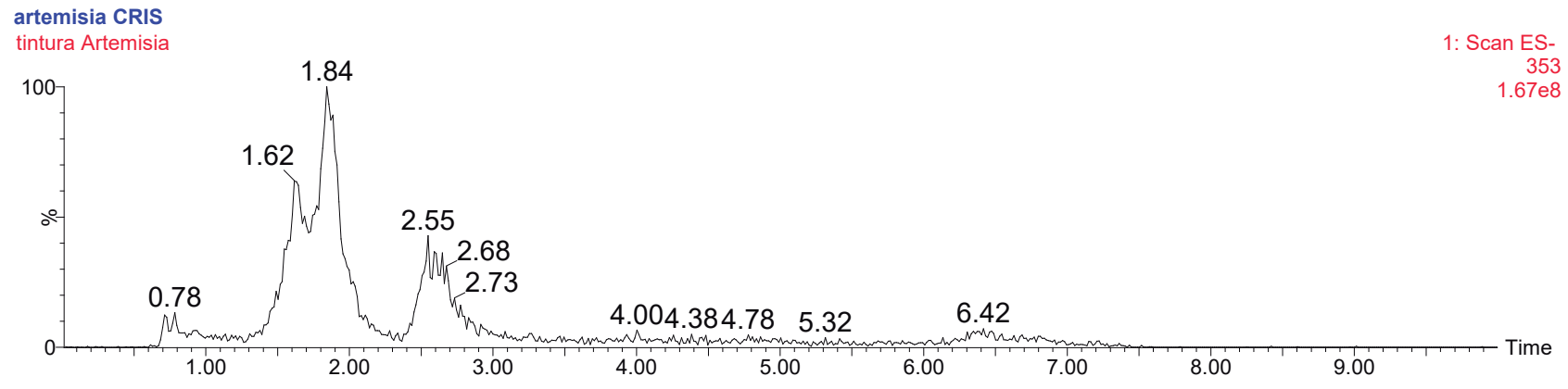

\section{Figura 4C.}

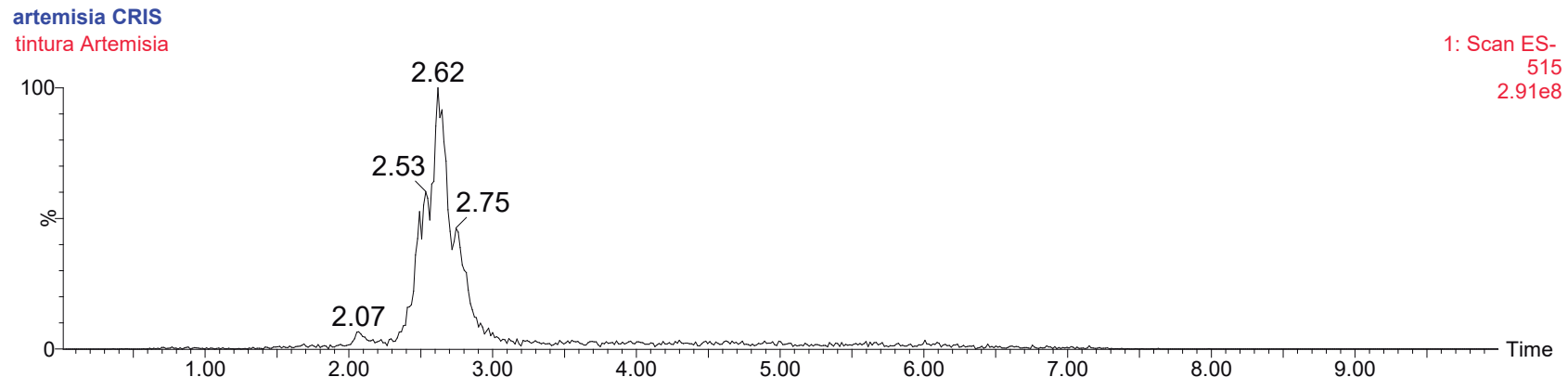

O volume de injeção foi de $120 \mu$ l, detecção em 320 $\mathrm{nm}$, consistindo de um gradiente iniciando-se com $100 \%$ de A, 5 minutos $80 \%$ de A, 21 minutos $70 \%$ de A e 34 minutos $100 \%$ de $\mathrm{A}$, o fluxo é $0,8 \mathrm{ml} / \mathrm{minuto}$, tempo de corrida 45 minutos.

A cromatografia (HPLC) comprova a existência do ácido clorogênico em 16,87 minutos e de ácidos cafeoilquinicos entre 28 e 35 minutos, aproximadamente (Figura 5).

\section{Cultura, tratamento e produção de citocinas}

Após a leitura das placas do teste de ELISA, obtivemos os seguintes resultados:

A figura 6 indica um aumento progressivo da produção da IL-4 a partir de $30 \mu \mathrm{L}$ de extrato de $A$. vulgaris, o que, segundo Oliveira CM et al [16], pode ter como consequência o aumento de uma resposta anti-inflamatória. Enquanto a figura 7 mostra uma produção diminuída de IL-6 frente ao estímulo com LPS, a partir do tratamento com $15 \mu \mathrm{L}$ do extrato, o que pode ser precedente para uma menor resposta pró-inflamatória. 
Figura 5.

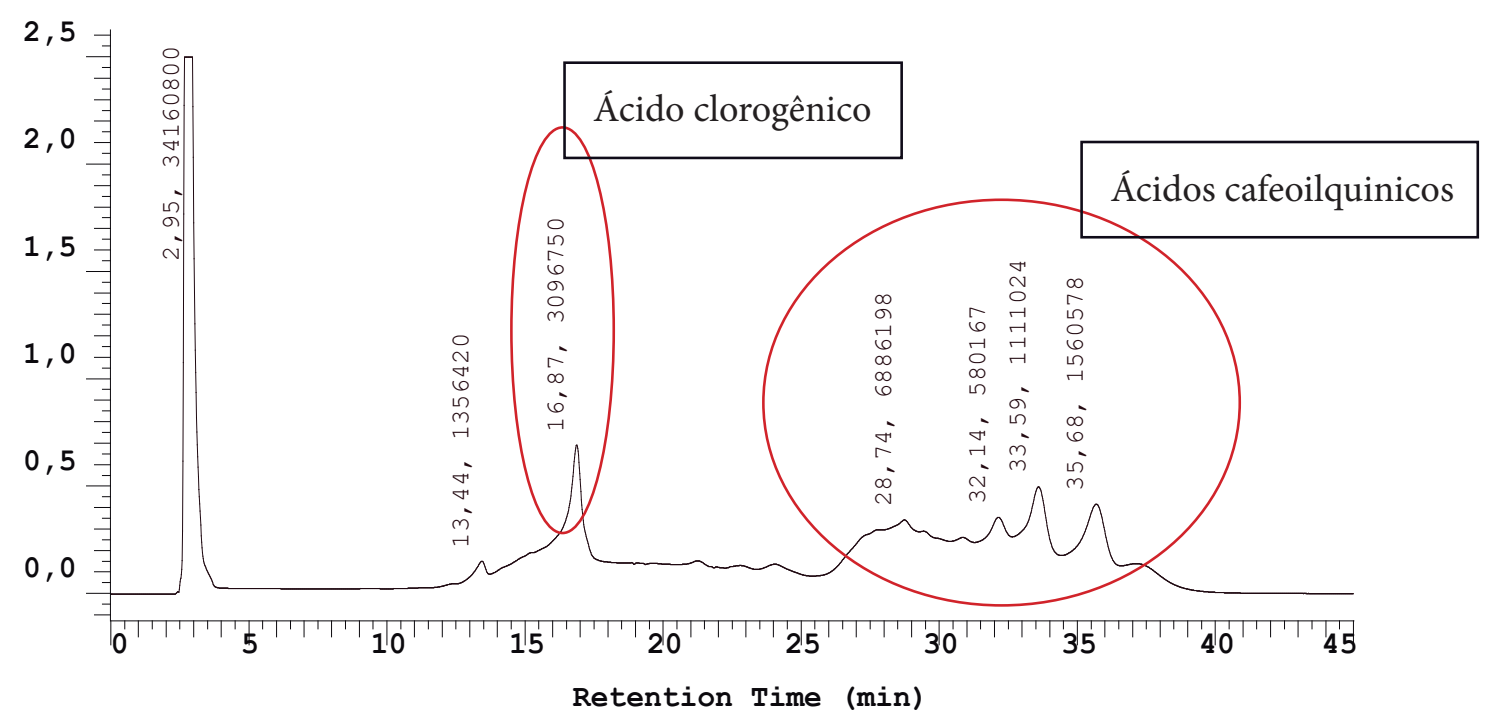

Figura 6, 7 .

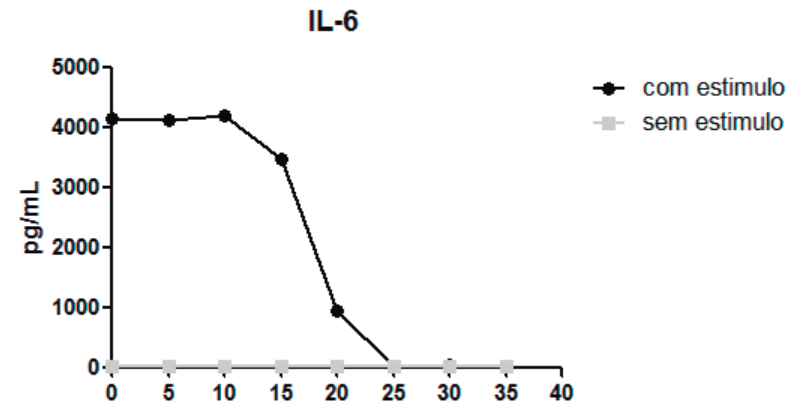

Figura 6

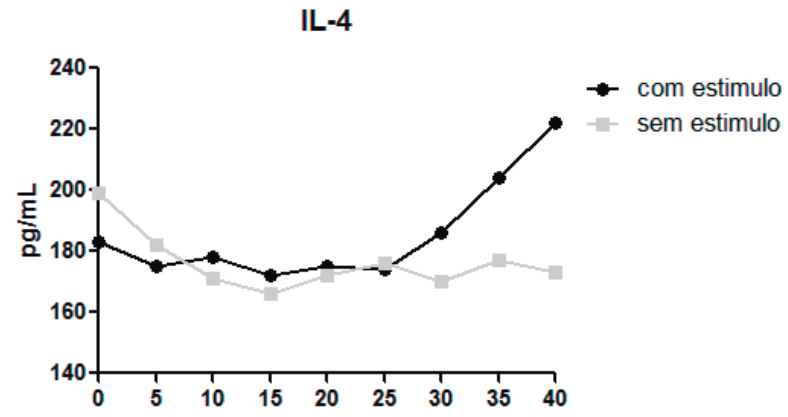

Figura 7

Figuras 6 e 7. Gráficos (pg/mL x volume em $\mu \mathrm{L}$ ) gerados a partir de imunoensaio (ELISA), demonstrando a atividade do EB de A.vulgaris na síntese de Interleucina (IL) sobre a linhagem celular RAW. A) IL-4. B) IL-6.

\section{Conclusão}

A partir dos resultados apresentados tanto da cromatografia liquida, quanto da produção de interleucinas, podemos sugerir que o extrato de Artemisia vulgaris possui atividade moduladora referente a IL-4 e IL-6, porém foram necessários mais estudos para a identificação de qual, ou quais compostos são responsáveis por essa modulação, uma vez que há grande quantidade de ácidos cafeoilquinicos e clorogênicos, além da artemisinina encontrada no extrato.

\section{Referências}

1. MAJDALAWIEH AF, FAYYAD MW. Immu- nomodulatory and anti-inflammatory action of Nigella sativa and thymoquinone: A comprehensive review. Int Immunopharmacol. 2015;28(1):295-304.

2. MOHAMED SIA, JANTAN I, HAQUE MA. Naturally occurring immunomodulators with antitumor activity: An insight on their mechanisms of action. Int Immunopharmacol. 2017;50:291-304.

3. CHANOTIYA C, SINGH S. LOW Molecular Weight Volatiles in Western Himalayan Artemisia. 2012. https://www.researchgate.net/ publication/291265126_Low_Molecular_Weight_ Volatiles_in_Western_Himalayan_ArtemWoSa.

4. GUETAT A, AL-GHAMDI FA, OSMAN AK. The genus Artemisia L. in the northern region of Saudi Arabia: essential oil variability and antibacteri- 
al activities. Nat Prod. Res. 2017;31(5):598-603.

5. CHEN J, WANG X, LIU C. Anti-tumour effects of polysaccharides isolated from Artemisia annua $\mathrm{L}$ by inducing cell apoptosis and immunomodulatory anti-hepatoma effects of polysaccharides. Afr. J Tradit Complement Altern Med. 2014;11(1):15-22.

6. HUNT S, STEBBINGS S, MCNAMARA D. An open-label six-month extension study to investigate the safety and efficacy of an extract of Artemisia annua for managing pain, stiffness and functional limitation associated with osteoarthritis of the hip and knee. N Z Med J. 2016;129(1444):97-102.

7. BAO X, YUAN H, WANG C, LIU J, LAN M. Antitumor and immunomodulatory activities of a polysaccharide from Artemisia argyi. Carbohydr Polym. 2013;98(1):1236-43.

8. HATZIIEREMIA S, GRAY AI, FERRO VA, PAUL A, PLEVIN R. The effects of cardamonin on lipopolysaccharide-induced inflammatory protein production and MAP kinase and NFkappaB signalling pathways in monocytes/macrophages. $\mathrm{Br} \mathrm{J}$ Pharmacol. 2006;149(2):188-98.

9. KREBS S, OMER TN, OMER B. WORMWOOD (Artemisia absinthium) suppresses tumour necrosis factor alpha and accelerates healing in patients with Crohn's disease - A controlled clinical trial. Phytomedicine. 2010;17(5):305-9

10. LEE HG, KIM H, OH WK, YU KA, CHOE YK, AHN JS. Tetramethoxy hydroxyflavone p7F downregulates inflammatory mediators via the inhibition of nuclear factor kappaB. Ann N Y Acad Sci.

11. AMAT $N$, UPUR $H, B L A Z E K O V I C ~ B$. In vivo hepatoprotective activity of the aqueous extract of Artemisia absinthium L. against chemically and immunologically induced liver injuries in mice. J Ethnopharmacol. 2010;131(2):478-84.

12. TEMRAZ A, EL-TANTAWY WH. Characterization of antioxidant activity of extract from Artemisia vulgaris. Pak J Pharm Sci. 2008;21(4):321-6.

13. FERREIRA MLC. Polissacarídeos solúveis de folhas de Artemisia absinthium e Artemisia vulgaris: isolamento, caracterização e efeitos sobre células THP-1. fevereiro 2014.

14. KUCUKBOYACI N, ERDOGAN ORHAN I, MURAT K, NEZAKET A, BANI B. Determination of Artemisinin in Selected Artemisia L. Species of Turkey. by Reversed Phase HPLC2007.

15. KHAIRUL IKRAM N, BEYRAGHDAR KASHKOOLI A, VITHAKSHANA PERAMUNA A,
KROL A, BOUWMEESTER H, SIMONSEN HT. Stable Production of the Antimalarial Drug Artemisinin in the Moss Physcomitrella patens2017.

16. OLIVEIRA CMBD, SAKATA RK, ISSY AM, GEROLA LR, SALOMÃO R. Citocinas e dor. Revista Brasileira de Anestesiologia. 